\title{
Indirect measurement of the magnetocaloric effect using a novel differential scanning calorimeter with magnetic field
}

\author{
Jeppesen, Stinus; Linderoth, Søren; Pryds, Nini; Kuhn, Luise Theil; Jensen, Jesper Buch
}

Published in:

Review of Scientific Instruments

Link to article, DOI:

$10.1063 / 1.2957611$

Publication date:

2008

Document Version

Publisher's PDF, also known as Version of record

Link back to DTU Orbit

Citation (APA):

Jeppesen, S., Linderoth, S., Pryds, N., Kuhn, L. T., \& Jensen, J. B. (2008). Indirect measurement of the magnetocaloric effect using a novel differential scanning calorimeter with magnetic field. Review of Scientific Instruments, 79(8), 083901. https://doi.org/10.1063/1.2957611

\section{General rights}

Copyright and moral rights for the publications made accessible in the public portal are retained by the authors and/or other copyright owners and it is a condition of accessing publications that users recognise and abide by the legal requirements associated with these rights.

- Users may download and print one copy of any publication from the public portal for the purpose of private study or research.

- You may not further distribute the material or use it for any profit-making activity or commercial gain

- You may freely distribute the URL identifying the publication in the public portal 


\title{
Indirect measurement of the magnetocaloric effect using a novel differential scanning calorimeter with magnetic field
}

\author{
S. Jeppesen, a) S. Linderoth, N. Pryds, L. Theil Kuhn, and J. Buch Jensen \\ Fuel Cells and Solid State Chemistry Department, Risф DTU, Technical University of Denmark, \\ DK-4000 Roskilde, Denmark
}

(Received 10 April 2008; accepted 20 June 2008; published online 5 August 2008)

\begin{abstract}
A simple and high-sensitivity differential scanning calorimeter (DSC) unit operating under magnetic field has been built for indirect determination of the magnetocaloric effect. The principle of the measuring unit in the calorimeter is based on Peltier elements as heat flow sensors. The high sensitivity of the apparatus combined with a suitable calibration procedure allows very fast and accurate heat capacity measurements under magnetic field to be made. The device was validated from heat capacity measurements for the typical DSC reference material gallium (Ga) and a $\mathrm{La}_{0.67} \mathrm{Ca}_{0.33} \mathrm{MnO}_{3}$ manganite system and the results were highly consistent with previous reported data for these materials. The DSC has a working range from 200 to $340 \mathrm{~K}$ and has been tested in magnetic fields reaching $1.8 \mathrm{~T}$. The signal-to-noise ratio is in the range of $10^{2}-10^{3}$ for the described experiments. Finally the results have been compared to results from a Quantum Design® physical properties measuring system. The configuration of the system also has the advantage of being able to operate with other types of magnets, e.g., permanent magnets or superconducting coils, as well as the ability to be expanded to a wider temperature range. (C) 2008 American Institute of Physics.
\end{abstract}

[DOI: $10.1063 / 1.2957611]$

\section{INTRODUCTION}

The heat capacity of a material as a function of temperature is important for the determination of the thermodynamic properties of a material. The use of calorimetry to investigate the magnetocaloric effect (MCE) is an important tool in the development and testing of new materials, e.g., magnetic refrigeration. Magnetic refrigeration is an emerging technology that has the potential for high energy efficiency. ${ }^{1,2}$ Recent research and development of magnetic refrigerants have demonstrated large magnetocaloric effects in the vicinity of room temperature in several materials ${ }^{3,4}$ and the need for fast and reliable characterization techniques of the MCE is increasing. As pointed out by Pecharsky and Gschneidner, ${ }^{5}$ calorimetry provides a method to minimize the systematic errors in the determination of the isothermal magnetic entropy change from heat capacity data as a complement to magnetization data.

Experimentally, the heat capacity is measured by calorimetry. Although a variety of commercially available instruments are used for this purpose, the ability to conduct thermal measurements as a function of applied magnetic field is still quite rare. ${ }^{6-9}$ Apart from the obvious advantage of minimizing systematic errors by using heat capacity measurements, the calorimetric approach also provides a fast way to obtain information on magnetocaloric effects as well as structural changes and charge ordering. ${ }^{10}$

Different methods to determine the MCE have been suggested in literature. $5,7,11$

\footnotetext{
${ }^{a)}$ Author to whom correspondence should be addressed. Tel.: +454677 5774. FAX: +45 4677 5858. Electronic mail: stinus.jeppesen@ risoe.dk.
}

(1) Direct measurement. Measurements of the adiabatic temperature change, $\Delta T_{\text {ad }}$, by moving the sample in and out of a magnetic field region and recording the changes in the temperature.

(2) Indirect measurement. Magnetization method: by determining the magnetization curves at various temperatures, the magnetic contribution to the entropy change, $\Delta S_{M}$, can be extracted.

(3) Indirect measurement. Calorimetric method: by measuring specific heat capacity as a function of temperature at various magnetic field strengths.

The second method is the most commonly used, while methods 1 and 3 require specialized equipment.

Clearly, the determination of the thermodynamic properties of materials, which exhibit a MCE, requires a reliable and accurate determination of the heat capacity of a sample under magnetized conditions. By knowing the heat capacity, $C$, as a function of temperature, $T$, and as a function of applied magnetic field, $H$, it is possible to calculate the isothermal magnetic entropy change $\Delta S_{M}$ :

$$
\begin{aligned}
& \Delta S_{M}(T)=S(T)_{H_{F}}-S(T)_{H_{I}}, \\
& S(T)_{H_{I, F}}=\int_{0}^{T} \frac{C(T)_{H_{I, F}}}{T} d T+S_{0, H_{I, F}},
\end{aligned}
$$

where the subscripts $I$ and $F$ denote initial and final states. $S_{0}$ is the zero temperature entropy normally assumed to be zero. ${ }^{1}$

In this paper, we present a simple and very accurate differential scanning calorimeter (DSC) for the determination of the specific heat of samples under magnetic field. 


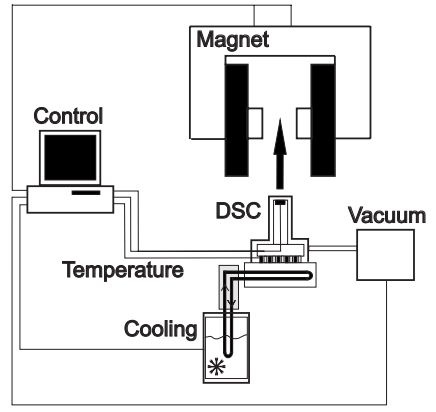

FIG. 1. Schematic illustration of the DSC setup. In this setup the temperature of the sample (heating and cooling) is controlled by a Peltier element with an additional separate cooling unit. The DSC can be operated with and without magnetic field. The running parameters of the DSC are controlled from a computer. In order to ensure a complete isolation of the sample from the surroundings, the sample is also kept in vacuum using a turbopump system which is coupled to the DSC unit.

\section{EXPERIMENTAL DETAILS}

\section{A. Construction of the calorimeter}

When designing the calorimeter, a large effort was devoted to optimization according to these criteria:

(1) accurate heat capacity measurements under magnetic field,

(2) determination of the MCE must be optimized around room temperature, and

(3) the measurement should be performed in near-adiabatic conditions.

A description of the calorimeter is schematically depicted in Fig. 1. The calorimetric block is a solid copper cylinder of high thermal conductivity. This configuration allows for the use of any standard laboratory electromagnet, a superconducting coil magnet, or, alternatively, a permanent magnet. The temperature is measured to within $\pm 0.1 \mathrm{~K}$ by means of a platinum resistance thermometer placed in the DSC cavity. The Peltier element acts like 16 thermocouples placed thermally in parallel and electrically in series, which puts the calorimeter into the family of Calvet calorimeters. ${ }^{12}$ This idea to use Peltier elements as heat flow sensors was proposed by Plackowski et al. $^{7}$ and has the advantage of obtaining highly reproducible data with high sensitivity in a very inexpensive manner. As opposed to Plackowski et al. and other types of insets to superconducting magnets ${ }^{6-9}$ this setup was designed to be more flexible in the adaption into the applied magnetic field. By keeping the measuring unit as a)

b)

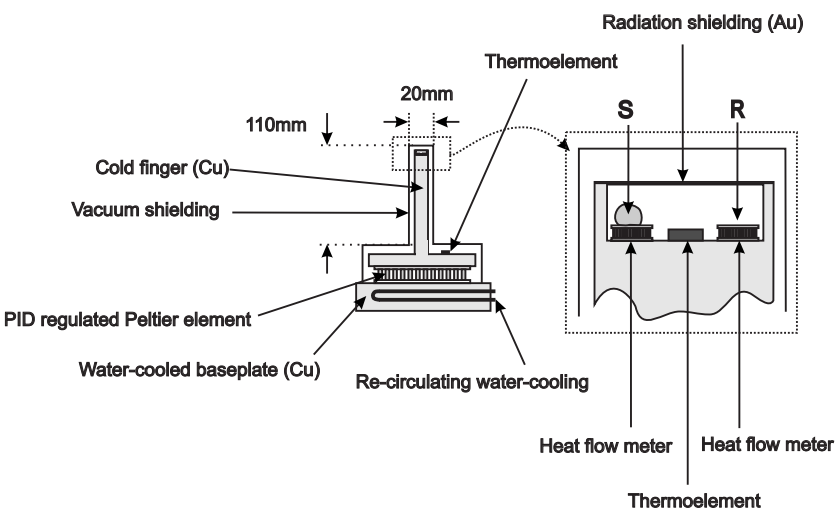

FIG. 2. Schematic of the measuring unit. (a) The complete DSC unit and (b) a close-up of the sample holder. (a) The temperature is controlled by a water-cooled baseplate mounted with a Peltier element. The Peltier element is PID controlling the temperature of the cold finger by a LABVIEW $®$ interface. The thermoelement for the PID control is placed distant to the measuring unit to level out temperature fluctuations caused by the PID control, and the sample temperature is then logged close by the sample (s) and reference (r) by another thermoelement. The two small Peltier elements marked as sample (s) and reference (r) are independently used as heat flow sensors for measuring of the sample and the reference to provide a differential signal.

small as $20 \mathrm{~mm}$ in diameter and the cold finger reaching 110 $\mathrm{mm}$, this setup can be adapted into various sorts of magnets including electromagnets, which have a much lower running cost than the superconducting magnets. Furthermore the temperature control was optimized to have maximum stability in the room-temperature range contrary to temperature controls heating from liquid nitrogen or helium temperatures. The cavity is surrounded by a cover, which ensures thermal homogeneity and near-adiabatic conditions via thermal isolation from the environment. The design of the temperature control system was based on the ability to choose the temperature range by a secondary cooling liquid (see Fig. 1) in order to obtain maximum temperature stability during the measurements. The temperature, process control, and the data acquisition are controlled from a LABVIEW interface.

A detailed scheme of the cavity is shown in Fig. 2 and the basic design parameters are given in Table I. In order to minimize heat losses to the surroundings the calorimeter unit is placed in a vacuum chamber equipped with a gold radiation reflection shielding. The DSC signal in the cavity is acquired by two differentially connected heat flow sensors, which represent the sample $(S)$ and the reference $(R)$. In the

TABLE I. Design parameters for the experimental setup.

\begin{tabular}{lcc}
\hline \hline \multicolumn{1}{c}{ Element } & Dimension $(\mathrm{mm})$ & Specification \\
\hline $\begin{array}{l}\text { Sensor Peltier elements } \\
\text { Temperature control Peltier element }\end{array}$ & $3 \times 4$ & Supercool PE-018-03-09 \\
Cold finger & $62 \times 62$ & Supercool PE-127-20-15 \\
$\begin{array}{l}\text { Thermoresistor element } \\
\text { Power supply }\end{array}$ & $110 \times 20^{\phi}$ & Copper \\
$\begin{array}{l}\text { Multimeter } \\
\text { Hall sensor } \\
\text { Grease for mounting of the sample }\end{array}$ & $2 \times 3$ & Pt100 element $100 \Omega$ \\
\hline \hline
\end{tabular}


present setup the samples were mounted directly on the upper surface of the sample holder without the use of a pan, therefore, in order to ensure a good thermal contact thermal grease (Apiezon ${ }^{\circledR} \mathrm{H}$-grease) is used. The thermal grease was investigated for a possible phase transition well below the operating temperature range of the DSC, and no visible indication of a phase transition was found in this range. This observation combined with the fact that a very small amount of grease $(\sim 0.1 \mathrm{mg})$ was used allowed us to treat the grease as part of the background. The heat capacity of the grease is subtracted from the sample signal by including the grease used to mount the sample, when measuring the empty sample holder in the addenda measurement. The cavity temperature is simultaneously recorded by a resistance temperature detector which is situated in the proximity of the sample/reference (Thermoelement) [Fig. 2(b)]. A signal from a second sensor is used as feedback signal for the temperature proportional-integral-derivative (PID) controller [Fig. 2(a)].

Since the operation temperature range of this equipment was designed to be around room temperature, it was sufficient to control the temperature in the range of 200-340 K. The temperature range of this device can be expanded in the future to lower or/and higher temperatures.

\section{B. Data acquisition}

When heat passes through a Peltier element it generates a voltage drop $U_{p}$, which is proportional to the heat flow $Q_{p}$ passing through the specific element to the heat sink:

$$
U_{p}=\frac{S}{k} Q_{p},
$$

where $S$ is the Seebeck coefficient and $k$ is the thermal conductance of the Peltier element. ${ }^{7}$ At constant external conditions (temperature, pressure, and magnetic field) $S / k$ is constant and $U_{p}$ can therefore be used directly as a measure of the heat flow. The heat capacity $C_{p}$

$$
C_{p}(T)=\frac{Q_{p}}{\dot{T}_{s}}=\frac{k}{S} \frac{U_{p}}{\dot{T}_{s}}
$$

is then derived by the classical three-step procedure ${ }^{12}$ from three successive temperature scans of the sample: (1) scan of the sample, (2) scan of the reference sample (for the calibration of the heat capacity we have used copper $(99.999 \% \mathrm{Cu})$ as a reference material ${ }^{12,13}$ ), and (3) scan of an empty sample holder for determining the background signal. As described above the background signal includes the amount of Apiezon $\mathrm{H}$-grease used to mount the sample subsequently. During these scans the temperature is ramped at a constant rate. Keeping the experimental conditions identical, i.e., sample weight and heating/cooling rates, in all three scans the heat capacity can then be derived point by point for a range of temperatures $^{2}$

$$
C_{p, \text { sample }}(T)=\frac{U_{\text {sample }}(T)-U_{0}(T)}{U_{\text {ref }}(T)-U_{0}(T)} \frac{m_{\text {ref }}}{m_{\text {sample }}} C_{p, \text { ref }}(T) .
$$

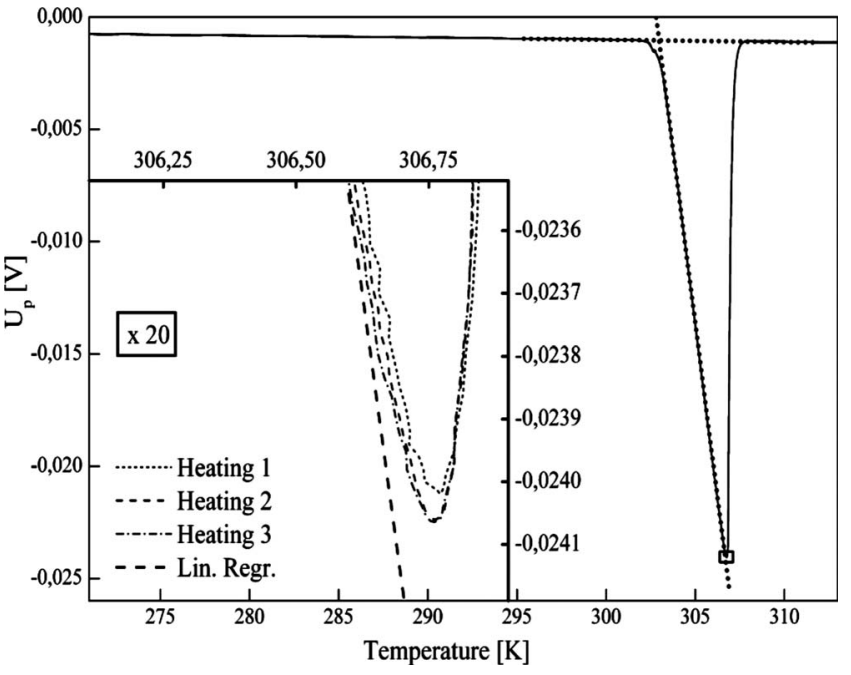

FIG. 3. Temperature calibration using the onset melting point $(303.05 \mathrm{~K})$ of gallium as reference material. Inserted figure shows the peak of the melting point magnified 20 times.

\section{Calibration}

The temperature measured in the setup has been calibrated by using the onset melting point of gallium (303.05 K) (Ref. 14) with high purity (99.999\%). The onset of the melting temperature used for the calibration is determined by the crossing point of the base level and slope of the peak during heating as shown in Fig. 3. The base level and the slope of the peak are determined by linear regression of the data below and above the melting peak and the linear range of the melting peak between 303.5 and $306.5 \mathrm{~K}$, respectively. The temperature calibration is only valid for a specific heating rate, and in our experiments, we kept the heating rate constant at $1 \mathrm{~K} / \mathrm{min}$ and the sample and reference of comparable sizes.

Besides the temperature calibration Fig. 3 demonstrates the excellent reproducibility of the measurements. In this case an endothermic process within the sample is recorded and illustrates the melting behavior of the material. Changes in ramping rate, heat loss, and thermal conductance would show up in the position of the melting peak. The very good reproducibility is illustrated by the inset, where three successive runs are shown. Furthermore, the melting enthalpy of gallium has been calculated and compared to values from the National Institute of Standards and Technology (NIST). The enthalpy of melting given by the NIST database ${ }^{15}$ is 80.097 $\mathrm{J} / \mathrm{g}$ in a $99.9999 \%$ pure Ga standard sample, whereas we calculated the enthalpy from our heat capacity data to be $80.8 \pm 0.2 \mathrm{~J} / \mathrm{g}$ in a $99.999 \%$ pure Ga sample, which implies a deviation of less than $1 \%$ including deviations due to differences in purity.

\section{RESULTS}

Since this setup was built for the purpose of characterizing the heat capacity of magnetocaloric materials as function of temperature and applied magnetic field, the sensitivity of the DSC needs to be tested on a much weaker signal e.g., from a magnetic phase transition. The magnetic phase transition from the ferromagnetic (FM) to paramagnetic (PM) 


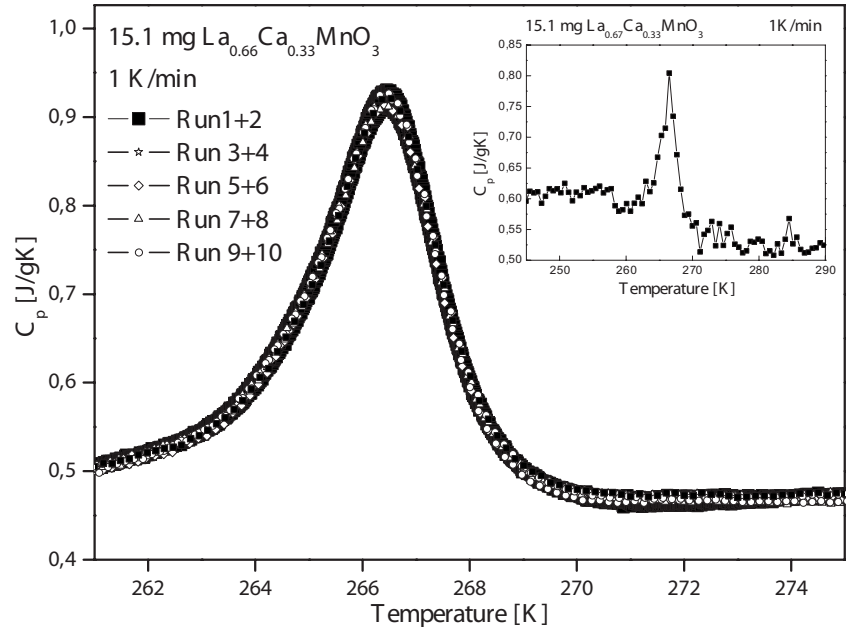

FIG. 4. Heat capacity as function of temperature in ten successive runs on the same $15.1 \mathrm{mg} \mathrm{La}{ }_{0.67} \mathrm{Ca}_{0.33} \mathrm{MnO}_{3}$ sample in zero magnetic field obtained by the new calorimeter. The inset shows heat capacity data of the same sample obtained in a Seiko DSC-120 commercial DSC setup.

state in the $\mathrm{La}_{0.67} \mathrm{Ca}_{0.33} \mathrm{MnO}_{3}$ system represents a signal, which is more than 50 times weaker than the melting of gallium. $\mathrm{La}_{0.67} \mathrm{Ca}_{0.33} \mathrm{MnO}_{3}$ (LCMO) was chosen since it is considered to be one of the possible candidate refrigerants in the field of room-temperature magnetic refrigeration and has a transition just around $267 \mathrm{~K}$ (Ref. 16) depending on the exact way of synthesis and heat treatment.

Figure 4 shows the results from ten successive runs on a $15.1 \mathrm{mg}$ LCMO sample and heating/cooling rate of $1 \mathrm{~K} / \mathrm{min}$. The signal due to the FM to PM transformation is measured to be only around $0.3 \mathrm{mV}$, whereas the signal from the melting of gallium was of the order $20 \mathrm{mV}$, i.e., the FM to PM signal is nearly two orders of magnitude smaller. The reproducibility of the signal and an excellent signal-to-noise ratio is, however, upheld, which demonstrates very well the capabilities of this measuring technique and the setup. The characteristic "lambda shape" of the phase transition is also clearly recognized.

The signal-to-noise ratio of the new DSC developed in our laboratory can be estimated by defining a noise band covering all measured data values in a linear part of the data set. This noise band includes the electrical noise of the measurement as well as the main contribution to the noise, namely, fluctuations in the signal derived from thermal fluctuation during the heating/cooling experiment. The width of the noise band can then be evaluated with the signal strength in a given phase transition investigated at a given heating rate. The width of the noise band has been estimated to be maximum $2.5 \mu \mathrm{V}$ over a $7 \mathrm{~K}$ range during heating at 1 $\mathrm{K} / \mathrm{min}$. With signal strength similar to the magnetic phase transition peak shown in Fig. 4 peaking around $0.3 \mathrm{mV}$, this will lead to a signal-to-noise ratio above 100/1. The much stronger signal from the melting of Ga shown in Fig. 3 is exceeding $20 \mathrm{mV}$ at the peak, leading to a signal-to-noise ratio of more than $800 / 1$ in that experiment. A zero-field heat capacity measurement of the same sample (Fig. 4 inset) measured in a commercial DSC setup (Seiko 120-DSC) led to a signal-to-noise ratio of less than $10 / 1$ for the same sample and experiment conditions.

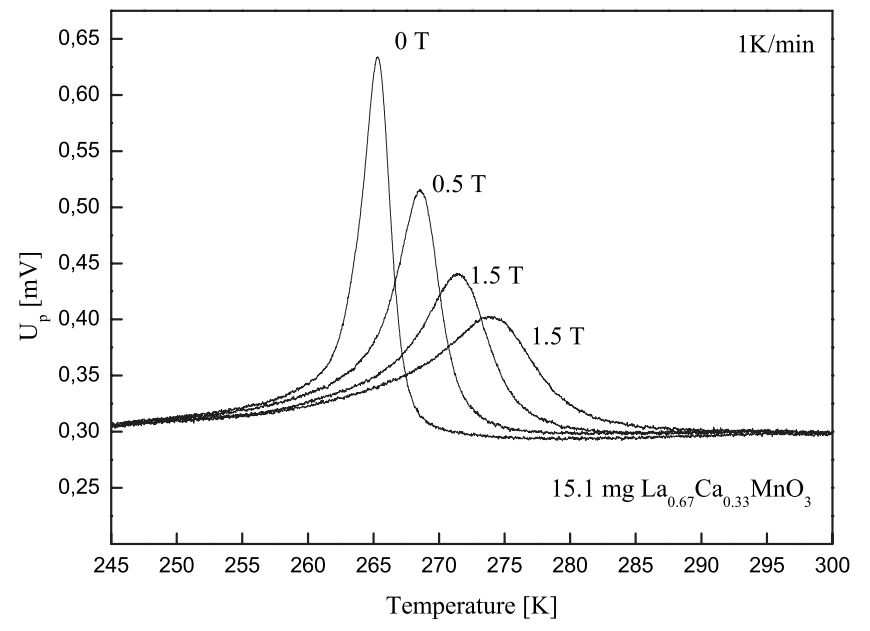

FIG. 5. The $15.1 \mathrm{mg} \mathrm{La}{ }_{0.67} \mathrm{Ca}_{0.33} \mathrm{MnO}_{3}$ sample measured in four different magnetic fields with the new calorimeter.

Sample sizes between 10 and $100 \mathrm{mg}$ are typically used in conventional DSC measurements depending on the exact material and experiment. As demonstrated when comparing the results in Fig. 4 and the inset in Fig. 4, the relative low weight when considering conventional DSC measurements of the $15.1 \mathrm{mg}$ LCMO sample results in a very high signalto-noise ratio in the setup described in this work, whereas to obtain equally good results in the commercial DSC it would be necessary to increase the sample size significantly or to change the heating rate.

On the results presented in Fig. 4 it furthermore becomes evident that even smaller samples would still give a satisfactory signal-to-noise ratios when measuring on FM to PM transitions.

The reproducibility and an excellent signal-to-noise ratio is hereby validated, confirming the results from the Ga runs (Fig. 3). Further, the enthalpy corresponding to the magnetic phase transition for the LCMO sample is more than 50 times smaller than that of the melting enthalpy of $\mathrm{Ga}$, and the reproducibility of the LCMO transition is therefore an important figure of merit for the performance of the new calorimeter.

The calorimeter has also been tested under applied magnetic fields. Figure 5 shows the influence of an applied magnetic field on the magnetic phase transition for the LCMO sample. As expected, the applied magnetic field changes the Curie temperature $\left(T_{C}\right)$ to higher temperatures and tends to broaden the shape of the phase transition. These results correspond well with the results obtained by direct measurements previously reported. ${ }^{17}$ The absolute comparison of our results and the results reported in the literature for the same material composition are not possible since the properties of these materials are very sensitive to the preparation procedure. e.g., heat treatment.

For a direct comparison with alternative measuring techniques the same ceramic material was measured in a Quantum Design ${ }^{\circledR}$ physical properties measuring system (PPMS). The results are shown in Fig. 6 and demonstrate an excellent agreement between the two techniques. The PPMS uses a relaxation technique and requires up to 40 min per measuring 


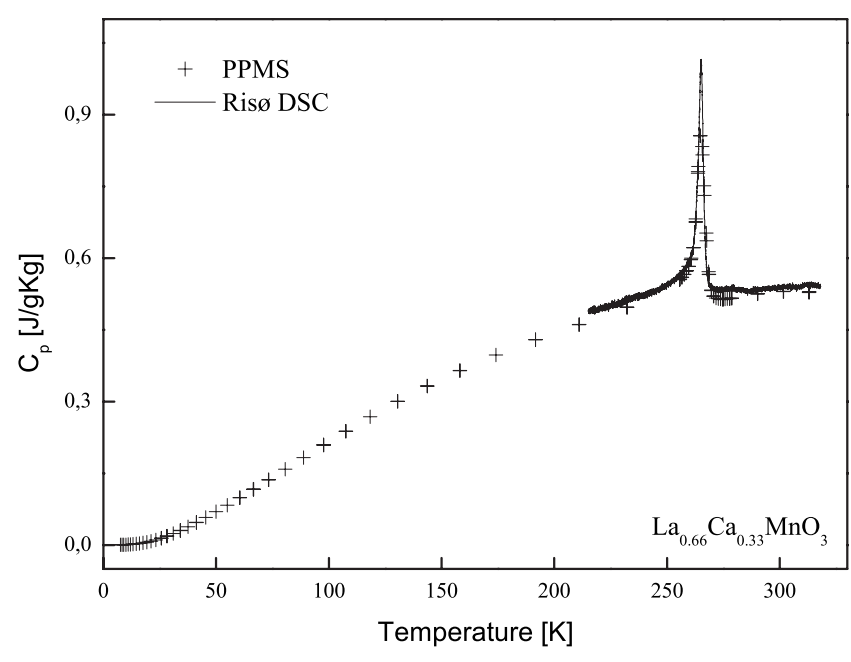

FIG. 6. $\mathrm{La}_{0.67} \mathrm{Ca}_{0.33} \mathrm{MnO}_{3}$ sample measured in a Quantum Design PPMS system and compared to the measurement from the DSC setup described in this article.

point, whereas scans with the Ris $\varnothing$ DSC is normally carried out with a scan rate of $1 \mathrm{~K} / \mathrm{min}$ and $2 \mathrm{~Hz}$ data logging frequency. This difference is also easily recognized in Fig. 6 where the data set from the Risø DSC shows much higher density of the data.

\section{CONCLUSION}

A novel DSC was constructed for the purpose of characterizing magnetocaloric materials in the near roomtemperature range (200-340 K). A significant improvement of the signal-to-noise ratio has been observed when compared to a conventional zero magnetic field DSC. Test results on pure $\mathrm{Ga}$ and $\mathrm{La}_{0.67} \mathrm{Ca}_{0.33} \mathrm{MnO}_{3}$ have been presented to demonstrate the performance of the setup. Test runs as a function of applied magnetic field demonstrate the ability of this device to operate correctly under applied magnetic fields up to $1.8 \mathrm{~T}$. The results from the DSC are in good agreement with results obtained in a commercial PPMS system from Quantum Design®.

\section{ACKNOWLEDGMENTS}

The authors acknowledge M. Christensen for carrying out the PPMS measurements in Department of Chemistry at the University of Aarhus, T. Henriksen for carrying out the programming of the LABVIEW interface, A.R. Dinesen for useful discussions during construction of the calorimeter, and A. Junod for helpful correspondence. For technical assistance Jørgen Geyti, Jens F.S Borchsenius, and Jørgen Poulsen are acknowledged.

${ }^{1}$ A. Tishin and Y. Spichkin The Magnetocaloric Effect and its Applications (IOP, Bristol, 2003).

${ }^{2}$ B. F. Yu, Q. Gao, B. Zhang, X. Z. Meng, and Z. Chen, Int. J. Refrig. 26, 622 (2003).

${ }^{3}$ K. A. Gschneidner, Jr., V. K. Pecharsky, and A. O. Tsokol, Rep. Prog. Phys. 68, 1479 (2005).

${ }^{4}$ E. Bruck, O. Tegus, D. T. C. Thanh, and K. H. J. Buschow, J. Magn. Magn. Mater. 310, 2793 (2007).

${ }^{5}$ V. K. Pecharsky and K. A. Gschneidner, J. Appl. Phys. 86, 565 (1999).

${ }^{6}$ V. K. Pecharsky, J. O. Moorman, and K. A. Gschneidner, Rev. Sci. Instrum. 68, 4196 (1997).

${ }^{7}$ T. Plackowski, Y. X. Wang, and A. Junod, Rev. Sci. Instrum. 73, 2755 (2002).

${ }^{8}$ J. Marcos, F. Casanova, X. Batlle, A. Labarta, A. Planes, and L. Manosa, Rev. Sci. Instrum. 74, 4768 (2003).

${ }^{9}$ M. Kuepferling, C. P. Sasso, V. Basso, and L. Giudici, IEEE Trans. Magn. 43, 2764 (2007).

${ }^{10}$ A. Szewczyk, M. Gutowska, B. Dabrowski, T. Plackowski, N. P. Danilova, and Y. P. Gaidukov, Phys. Rev. B 71, 224432 (2005).

${ }^{11}$ A. Szewczyk, M. U. Gutowska, K. Piotrowski, M. Gutowski, M. T. Borowiec, V. P. Dyakonov, V. L. Kovarskii, H. Szymczak, and L. Gladczuk, J. Phys.: Condens. Matter 10, 10539 (1998).

${ }^{12}$ G. W. H. Höhne, W. F. Hemminger, and H. J. Flammersheim, Differential Scanning Calorimetry (Springer, Berlin, 2003).

${ }^{13}$ G. Furukawa, W. Saba, and M. Reilly, Natl. Stand. Ref. Data Ser. 18, 1 (1968).

${ }^{14}$ K. J. Chau, K. M. Rieckmann, and A. Y. Elezzabi, Appl. Phys. Lett. 90, 131114 (2007).

${ }^{15}$ NIST Standard Reference Materials, 2005, https://srmors.nist.gov/tables/ pdf/203_5.pdf

${ }^{16}$ A. R. Dinesen, S. Linderoth, and S. Morup, J. Magn. Magn. Mater. 253, 28 (2002).

${ }^{17}$ A. R. Dinesen, "Magnetocaloric and magnetoresistive properties of $\mathrm{La}_{0.67} \mathrm{Ca}_{0.33}-x \mathrm{Sr}_{x} \mathrm{MnO}_{3}$," Ph.D. thesis, Ris $\emptyset$ National Laboratory, Technical University of Denmark, 2004. 